\title{
Phase-Change Materials: Vibrational Softening upon Crystallization and Its Impact on Thermal Properties
}

\author{
Toshiyuki Matsunaga,* Noboru Yamada, Rie Kojima, Shinichi Shamoto, Masugu Sato, \\ Hajime Tanida, Tomoya Uruga, Shinji Kohara, Masaki Takata, Peter Zalden, \\ Gunnar Bruns, Ilya Sergueev, Hans Christian Wille, Raphaël Pierre Hermann, \\ and Matthias Wuttig
}

Crystallization of an amorphous solid is usually accompanied by a significant change of transport properties, such as an increase in thermal and electrical conductivity. This fact underlines the importance of crystalline order for the transport of charge and heat. Phase-change materials, however, reveal a remarkably low thermal conductivity in the crystalline state. The small change in this conductivity upon crystallization points to unique lattice properties. The present investigation reveals that the thermal properties of the amorphous and crystalline state of phase-change materials show remarkable differences such as higher thermal displacements and a more pronounced anharmonic behavior in the crystalline phase. These findings are related to the change of bonding upon crystallization, which leads to an increase of the sound velocity and a softening of the optical phonon modes at the same time.

\section{Introduction}

Phase-change materials are characterized by a unique combination of properties including fast crystallization of the amorphous phase accompanied by a drastic change of electronic properties such as optical reflectance and electrical resistance. These attributes offer interesting challenges for our understanding of solids. At the same time, these properties also provide an exciting application potential in optical and electronic data storage..$^{[1]}$ Most materials presently used for optical recording are located on the pseudobinary line between $\mathrm{GeTe}$ and $\mathrm{Sb}_{2} \mathrm{Te}_{3} \cdot{ }^{\left[{ }^{[2]}\right.}$ These compounds are very promising for nonvolatile electronic memory devices, ${ }^{[3-5]}$ since their electrical resistance changes
Dr. T. Matsunaga

Materials Science and Analysis Technology Centre

Panasonic Corporation

3-1-1 Yagumo-Nakamachi, Moriguchi, Osaka 570-8501, Japan

E-mail: matsunaga.toshiyuki@jp.panasonic.com

Japan Synchrotron Radiation Research Institute

Hyogo, Japan

Dr. N. Yamada

Digital \& Network Technology Development Centre

Panasonic Corporation

Osaka, Japan

Japan Synchrotron Radiation Research Institute

Hyogo, Japan

Dr. R. Kojima

Digital \& Network Technology Development Centre

Panasonic Corporation

Osaka, Japan

Dr. S. Shamoto

Neutron Science Research Centre

Japan Atomic Energy Research Institute

Ibaraki, Japan

Dr. M. Sato, Dr. H. Tanida, Dr. T. Uruga, Dr. S. Kohara Japan Synchrotron Radiation Research Institute

Hyogo, Japan

DOI: 10.1002/adfm.201002274
Prof. M. Takata

Japan Synchrotron Radiation Research Institute

Hyogo, Japan

SPring-8/RIKEN

Hyogo, Japan

Department of Advanced Materials Science

School of Frontier Sciences

The University of Tokyo

Chiba, Japan

Dr. I. Sergueev

European Synchrotron Radiation Facility

38043 Grenoble, France

Dr. H. C. Wille

Deutsches Elektronen-Synchrotron

22607 Hamburg, Germany

Dr. R. P. Hermann

Inv. Prof., Jülich Centre for Neutron Science JCNS and Peter Grünberg Institut PGI

JARA-FIT, Forschungszentrum Jülich $\mathrm{GmbH}, 52425$ Jülich, Germany

Faculté des Sciences

Université de Liège

4000 Liège, Belgium

P. Zalden, G. Bruns, Prof. M. Wuttig

I. Physikalisches Institut (IA) and JARA-FIT

RWTH Aachen University

52056 Aachen, Germany 
significantly upon the phase transformation from amorphous to crystalline state. ${ }^{[6]}$ The class of materials between GeTe and $\mathrm{Sb}_{2} \mathrm{Te}_{3}$ is especially suited for application in memory cells because of their low thermal conductivity, ${ }^{[7]}$ which helps to decrease the electric power necessary to thermally cycle a memory cell from the crystalline to the amorphous state via melt-quenching.

To fully exploit the potential of phase-change materials, it is therefore crucial to understand the whole property portfolio which characterizes these materials. In recent years, significant progress has been achieved in realizing and understanding the unusual properties of phase-change materials, as summarized in Table 1. The pronounced optical contrast between the amorphous and crystalline phase, which is lacking in $\mathrm{sp}^{3}$-bonded semiconductors such as $\mathrm{Si}$ or GaAs, has been attributed to a change of bonding upon crystallization. ${ }^{[8-10]}$ While 'ordinary', i.e., saturated covalent bonds are employed in the amorphous phase, the crystalline structure is stabilized by resonance bonding, i.e., a superposition of configurations with saturated covalent bonds. This difference in bonding explains the strong contrast in optical reflectivity. ${ }^{[11]}$ The electrical resistivity also changes by several orders of magnitude upon crystallization. ${ }^{[6]}$ Resonance bonding helps to explain peculiar features in the electronic transport of the crystalline phase such as disorder-induced localization (Anderson localization). ${ }^{[12]}$ It is surprising, though, that the thermal conductivity of the amorphous and crystalline states behaves rather similarly in contrast to the pronounced changes observed upon crystallization for the optical and electrical properties. The metastable cubic phase of $\mathrm{Ge}_{2} \mathrm{Sb}_{2} \mathrm{Te}_{5}$, for example, has a thermal conductivity of approximately $0.44 \mathrm{~W} \mathrm{~K}^{-1} \mathrm{~m}^{-1}$ (see Supporting Information for details), only slightly higher than the corresponding value of the amorphous state (around $0.25 \mathrm{~W} \mathrm{~K}^{-1} \mathrm{~m}^{-1}$ ). ${ }^{[7,13-18]}$ Both the low value of the thermal conductivity in crystalline phase-change materials and the small difference between the amorphous and crystalline state are quite remarkable and point to unique lattice properties. Ordinary covalent semiconductors, even those consisting of heavy elements, show a significantly larger thermal

Table 1. Characteristic properties of phase-change materials such as $\mathrm{GeTe}$ and $\mathrm{Ce}_{2} \mathrm{Sb}_{2} \mathrm{Te}_{5}$ in both the amorphous and crystalline state. In the amorphous state, phase-change materials utilize covalent bonding and display co-ordination numbers which are essentially consistent with the 8-N rule (see Supporting Information for a more detailed discussion on the co-ordination number in amorphous phase-change materials). For the crystalline phase, on the contrary, there is a significant difference between $\mathrm{sp}^{3}$-bonded semiconductors and $\mathrm{p}$-bonded phase-change materials.

\begin{tabular}{|c|c|c|}
\hline & Amorphous & Crystalline \\
\hline & $\mathrm{sp}^{3}$ - and $\mathrm{p}$-bonded & p-bonded phase-change \\
\hline & systems like Si and & materials like $\mathrm{Ge}_{2} \mathrm{Sb}_{2} \mathrm{Te}_{5}$ \\
\hline & GeTe & (sp³-bonded systems) \\
\hline Bonding & covalent & resonant (covalent) \\
\hline \multirow[t]{2}{*}{ Short-range order } & $8-\mathrm{N}$ rule & higher co-ordination \\
\hline & & number (8-N rule) \\
\hline Thermal conductivity & low & low (high) \\
\hline Optical reflectivity & - & high contrast (small contrast) \\
\hline
\end{tabular}

conductivity in the crystalline state, like $\mathrm{Ge}\left(60 \mathrm{~W} \mathrm{~K}^{-1} \mathrm{~m}^{-1}\right)$, ${ }^{[19]}$ GaAs (45 W K $\mathrm{K}^{-1} \mathrm{~m}^{-120]}$ ), InSb (20 W K $\left.\mathrm{K}^{-1} \mathrm{~m}^{-1[21]}\right)$, and $\mathrm{GaSb}$ $\left(60 \mathrm{~W} \mathrm{~K}^{-1} \mathrm{~m}^{-1[21]}\right)$. For these materials, the thermal conductivity changes substantially upon crystallization. A typical example is germanium, which has a thermal conductivity of $12 \mathrm{~W} \mathrm{~K}^{-1} \mathrm{~m}^{-1}$ in the amorphous ${ }^{[22]}$ and $60 \mathrm{~W} \mathrm{~K}^{-1} \mathrm{~m}^{-1}$ in the crystalline phase. In the phase-change materials studied so far, crystallization, however, is only accompanied by a very modest increase of the thermal conductivity; less than a factor of two. This modest increase is not due to differences in the electrical conductivity of these two states. The electronic contribution to the observed thermal conductivity is significantly smaller than the contribution of the vibrational modes according to the WiedemannFranz law ${ }^{[21]}$ and can therefore be neglected in the following discussion. Until now, it has not been convincingly explained why this increase of the thermal conductivity upon crystallization is so low for phase-change materials. Hence it would be a significant step forward in our understanding if the unique thermal properties of crystalline phase-change materials could be disentangled. It would be even better if a microscopic model could be developed that can explain both the electrical and optical, as well as the thermal, properties. This model would be a breakthrough that would provide important guidelines for the design of new phase-change materials, for which lowering the power needed to locally melt the crystalline phase is one of the key concerns. A low thermal conductivity of the crystalline state helps in reducing thermal losses to the surroundings and hence lowers the required power to locally melt the material.

\section{Results}

In this manuscript, we present experimental results to unravel the origin of the thermal properties of phase-change materials. We start from the atomic arrangement of both the amorphous and the crystalline phase, which is investigated over a large range of temperatures by combining X-ray diffraction and X-ray absorption spectroscopy techniques. Details on sample preparation and on the experimental methods can be found in the Experimental Section and in the Supporting Information.

Figure 1 shows the fitting results from K-edge extended $\mathrm{X}$-ray absorption fine structure (EXAFS) spectra in blue, i.e., the bond lengths in the amorphous phase of $\mathrm{Ge}_{2} \mathrm{Sb}_{2} \mathrm{Te}_{5}$. All bond lengths that are deducible from the EXAFS data are evaluated and shown in Figure 1, where the good agreement of the Ge-Te bond length, obtained from the Ge edge, and the Te-Ge bond length, obtained from the Te edge, demonstrate the reliability of the data. Almost no temperature dependence is observed for the measured bond lengths. This result indicates that the atomic potentials are very harmonic, in striking contrast to the crystalline phase, where a pronounced reversible change of the local interatomic spacing upon heating is observed by X-ray diffraction. A pair distribution function (PDF) refinement has been performed to determine contributions from short and long $\mathrm{Ge}-\mathrm{Te}$ and $\mathrm{Sb}-\mathrm{Te}$ bonds. A strong temperature dependence of bond lengths is observed. This dependence is evidence for a weaker and a more anharmonic interaction potential in the crystalline state. Hence the EXAFS for the amorphous state and the XRD data for the crystalline state in Figure 1 provide 


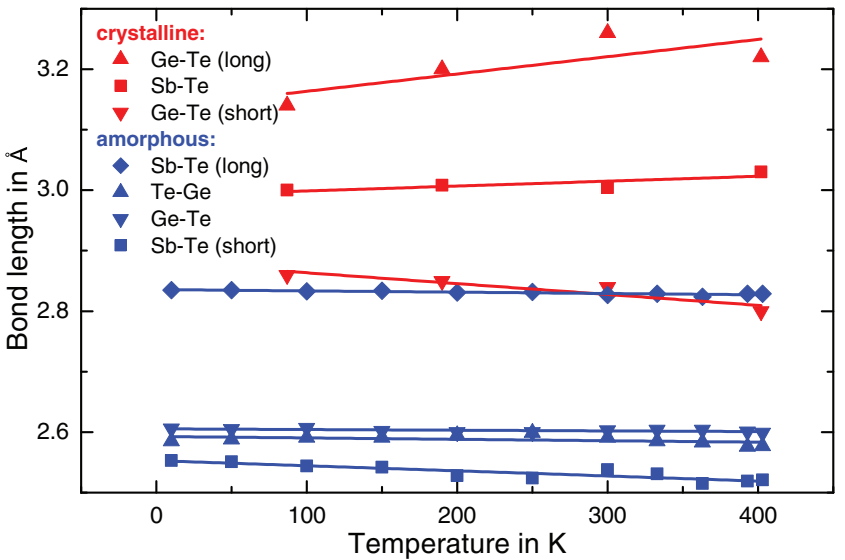

Figure 1. Anharmonic temperature dependence of bond lengths in the crystalline phase compared to the more harmonic amorphous phase. Local bond lengths of the amorphous phase (blue symbols) were determined by EXAFS analyses at all $\mathrm{K}$ absorption edges and different temperatures. The fits to different edges lead to consistent results (cf. Ge-Te vs. Te-Ge bond lengths). In the crystalline phase (red symbols), Ge-Te and Sb-Te bond lengths were obtained from the atomic parameters determined through PDF analyses. The low temperature dependence in the amorphous phase shows that this phase is dominated by harmonic interaction potentials while, on the contrary, the crystalline phase shows a strong temperature dependence, especially for the Ge-Te bonds. This result points to a strong anharmonic contribution to the interaction potential.

clear evidence for a change of interaction potential upon crystallization.

This indication of an anharmonic interaction potential in the metastable crystalline phase is also in line with the surprising temperature dependence of the mean-square-displacement parameter $\left\langle u^{2}\right\rangle$ derived from both EXAFS and diffraction data, which are both depicted in Figure 2, rescaled as $B=8 \pi^{2}<u^{2}>$. The atomic displacements in the metastable crystalline phase depend much more strongly on temperature than do those of the amorphous state. Again, this fact implies that the crystalline phase has a more anharmonic interaction potential. Yet, it has to be noted that two different techniques were employed to characterize the amorphous and crystalline states. While $\mathrm{XRD}$ probes the long-range order and quantifies disorder based on the displacement of atoms from the reflecting lattice planes, EXAFS reveals displacement parameters with a larger weighting on localized vibrational modes. ${ }^{[23]}$ Therefore, EXAFS is more sensitive to optical modes. To prove the vibrational softening upon crystallization it is hence necessary to apply the same experimental technique to characterize both phases.

This undertaking is not trivial since a technique is needed that provides both the necessary energy resolution and sufficient sensitivity to characterize the small amounts of material available. We hence decided to perform direct measurements of the density of phonon states, $g(E)$, in the amorphous and metastable crystalline phase of $\mathrm{GeSb}_{2} \mathrm{Te}_{4}$ using ${ }^{121} \mathrm{Sb}$ and ${ }^{125} \mathrm{Te}$ nuclear inelastic scattering (NIS). ${ }^{[24]}$ Because this method is not yet applicable to $\mathrm{Ge}$, we have chosen $\mathrm{GeSb}_{2} \mathrm{Te}_{4}$ over $\mathrm{Ge}_{2} \mathrm{Sb}_{2} \mathrm{Te}_{5}$ to analyze a larger fraction of the vibrational density of states. Since $\mathrm{GeSb}_{2} \mathrm{Te}_{4}$ and $\mathrm{Ge}_{2} \mathrm{Sb}_{2} \mathrm{Te}_{5}$ behave in a very similar way, we expect the same characteristic lattice dynamics and the same temperature dependence of the structure. The NIS data

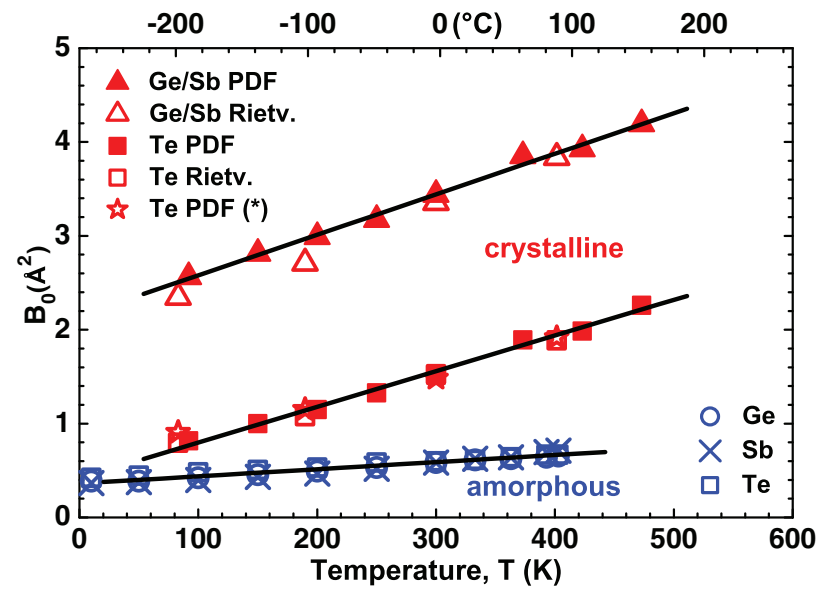

Figure 2. Temperature dependence of the atomic-displacement parameters $B$. Atomic-displacements parameters of $\mathrm{Ge}_{2} \mathrm{Sb}_{2} \mathrm{Te}_{5}$ have been obtained for the crystalline phase by X-ray diffraction Rietveld (open red marks) and PDF (full red marks) analyses. The amorphous phase was investigated by means of EXAFS measurements. The red stars show the parameters obtained by the PDF analysis, which assumes that $\mathrm{Ge} / \mathrm{Sb}$ and Te have identical amplitudes of thermal atomic vibrations and all atoms in the unit cell can freely move in any direction. The solid lines in the figure were obtained by least-squares analysis. Error bars are omitted for data with errors smaller than the marks.

give element-specific access to the purely dynamic atomic meansquare displacements, the force constants, and the elementspecific density of states. In analogy to the Debye-Waller factor, the mean-square displacements $\left\langle\Delta x^{2}\right\rangle=3 B / 8 \pi^{2}$, are directly related to the Lamb-Mössbauer fraction:

$f_{\mathrm{LM}}=\exp \left(-k^{2}\left\langle\Delta x^{2}\right\rangle\right)$

where $k$ is the wavevector of the nuclear resonant photons. Furthermore

$\left\langle\Delta x^{2}\right\rangle=\frac{\hbar^{2}}{2 M} \int_{0}^{\infty} g(E) E^{-1} \operatorname{coth}(\beta E / 2) d E$,

is obtained by the weighted minus-one moment of the density of phonon states, where $M$ is the mass of the atom and $\beta=1$ / $\left(\mathrm{k}_{\mathrm{B}} \mathrm{T}\right)$. In contrast, the force constant,

$F=\frac{M}{\hbar^{2}} \int_{0}^{\infty} g(E) E^{2} d E$,

is obtained from the second moment of the density of phonon states. The density of phonon states for $\mathrm{Sb}$ and $\mathrm{Te}$ in the amorphous and crystalline phase shown in Figure 3 (also see Table 2) reveals a remarkable change upon crystallization: Both a hardening of the low-energy acoustic phonons and a softening of the high-energy optical phonons are observed simultaneously. The first behavior is the characteristic hardening upon crystallization, which is frequently encountered and is accompanied by an increase in the speed of sound. The simultaneous lowering of the center of mass of the optical modes, however, is unique to our knowledge. 


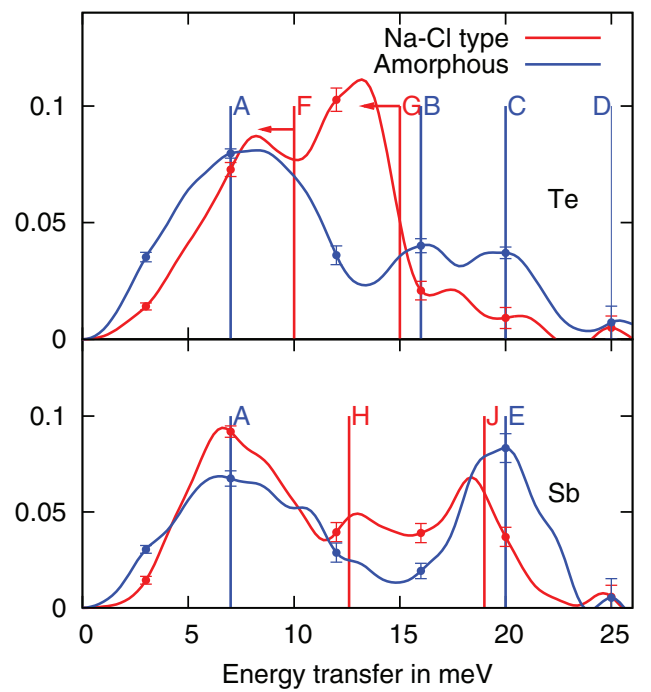

Figure 3. Elastic hardening and vibrational softening of optical modes: $\mathrm{Te}$ and Sb element-specific density of phonons states at $25 \mathrm{~K}$ in $\mathrm{GeSb}_{2} \mathrm{Te}_{4}$, with typical error bars for selected energies. Reported phonon energies for similar compounds are indicated by vertical lines and are summarized in Table 2. The peaks $F$ and $G$ are shifted to lower energies than for GeTe. The optical modes in both density functions shift to lower energies upon crystallization.

We have used these densities of states to obtain the purely dynamic displacement parameters and force constants (see Table 3). Displacement parameters are derived using the minus-one moment of the phonon densities and are weighted more towards the acoustic than the higher optical modes. This weighting results in the observation of an elastically harder crystalline phase for both $\mathrm{Sb}$ and Te atoms. Force constants, on the other hand, are calculated from the second moment of the phonon states and are governed by the optical modes. Therefore, force constants are lower in the crystalline phase, which thus reflects the unique vibrational softening upon crystallization. Also the Debye temperatures could be determined by fitting the Debye relation $g(w)=3 w^{2} / w_{D}^{3}$ to the low energy regime, i.e., to the acoustical modes of the phonon density of

Table 2. Phonon modes as indicated in Figure 3. The given energies are taken from published data.

\begin{tabular}{lccc}
\hline ID & Origin & Energy & Ref. \\
\hline A & Below A: Acoustic Te/Sb & $7 \mathrm{meV}$ & {$[53]$} \\
B & GeTe octa a) $^{\text {a }}$ & $16 \mathrm{meV}$ & {$[51]$} \\
C & GeTe octa $^{\text {a) }}$ & $20 \mathrm{meV}$ & {$[51]$} \\
D & GeTe tetra a) & $25 \mathrm{meV}$ & {$[51]$} \\
E & b) & $20 \mathrm{meV}$ & b) \\
F & GeTe octa (TO) & $10 \mathrm{meV}$ & {$[27]$} \\
G & GeTe octa (LO) & $15 \mathrm{meV}$ & {$[27]$} \\
H & $\mathrm{Eg}_{\mathrm{g}}$ (2) of $\mathrm{Sb}_{2} \mathrm{Te}_{3}$ (TO) & $12.6 \mathrm{meV}$ & {$[26]$} \\
J & $\mathrm{Al}_{\mathrm{g}}$ (2) of $\mathrm{Sb}_{2} \mathrm{Te}_{3}$ (LO) & $19 \mathrm{meV}$ & {$[26]$} \\
\hline
\end{tabular}

a) It is not possible to distinguish TO from LO modes in terms of energy scale in $\mathrm{GeTe} ;{ }^{[51]}$ b) No literature data on phonons of Ge-Sb bonds were found. This absence might be due to the instability of this alloy. ${ }^{[52]}$
Table 3. Elastic hardening and vibrational softening of optical modes upon crystallization is quantified by calculating ${ }^{[24]}$ the $\mathrm{Sb}$ and Te elementspecific isotropic-displacement parameters, $B$, and force constants, $F$, in the amorphous and crystalline phases of $\mathrm{GeSb}_{2} \mathrm{Te}_{4}$. Displacement parameters that are connected with elastic properties appear harder in the crystal, while force constants and optical modes appear softer in the crystalline phase.

\begin{tabular}{lccccc}
\hline & \multicolumn{2}{c}{$S b$} & & \multicolumn{2}{c}{$\mathrm{Te}$} \\
\cline { 2 - 3 } \cline { 5 - 6 } & $B\left[\AA^{2}\right]$ & $F\left[\mathrm{~N} \mathrm{~m}^{-1}\right]$ & & $B\left[\AA^{2}\right]$ & $F\left[\mathrm{~N} \mathrm{~m}^{-1}\right]$ \\
\hline Amorphous & $0.184(2)$ & $97(4)$ & & $0.198(2)$ & $84(4)$ \\
Na-Cl type & $0.166(2)$ & $72(4)$ & & $0.160(2)$ & $68(4)$ \\
\hline
\end{tabular}

states (PDOS). The fits reveal that crystallization leads to only a slight increase of the Debye temperature for the Te atoms from 120 to $151 \mathrm{~K}$ and from 131 to $153 \mathrm{~K}$ for the Sb atoms.

A detailed comparison of the densities of states with literature data gives further insight into the vibrational softening. The PDOS of the amorphous phase is dominated by a strong contribution of acoustic $\mathrm{Te}$ and $\mathrm{Sb}$ phonon states up to around $7 \mathrm{meV}$ (peak A in Figure 3). The Te atoms are involved to 65\% in Te-Sb and to $35 \%$ in Te-Ge bonds, ${ }^{[25]}$ with the latter showing a clear signature in peaks B and C. The shallow peak of tetrahedrally co-ordinated $\mathrm{Ge}$ could not be observed within the errors (no peak at $\mathrm{D}$ ). $\mathrm{Sb}$ atoms in amorphous $\mathrm{Ge}_{1} \mathrm{Sb}_{2} \mathrm{Te}_{4}$ are involved to $80 \%$ in $\mathrm{Sb}-\mathrm{Te}$ and to $20 \%$ in Sb-Ge bonds. ${ }^{[25]}$ Due to the distorted octahedral arrangement of $\mathrm{Sb}$ atoms in both the amorphous and the crystalline phase, the spectra for $\mathrm{Sb}$ do not differ significantly. Most dominantly, the center of mass around peak E shifts to lower energies and vibrational modes around $\mathrm{J}$ and $\mathrm{H}$ appear upon crystallization. Both the peaks at J and $\mathrm{H}$ perfectly match the values reported for calculations on crystalline $\mathrm{Sb}_{2} \mathrm{Te}_{3} .{ }^{[26]}$ These two optical modes are predominantly transversal and longitudinal, respectively, in character. The most striking effect of softening upon crystallization in $\mathrm{NaCl}$ type $\mathrm{Ge}_{1} \mathrm{Sb}_{2} \mathrm{Te}_{4}$, however, can be observed in the Te states where the hard modes at $\mathrm{B}$ and $\mathrm{C}$ are suppressed and a softer mode near $\mathrm{G}$ appears. For comparison, the modes in $\alpha-\mathrm{GeTe}^{[27]}$ are indicated by $\mathrm{F}$ and $\mathrm{G}$ and belong to transverse-optical (TO) and longitudinal-optical (LO) states, respectively. Both peak energies are reduced by about $2 \mathrm{meV}$ as compared to calculations at $0 \mathrm{~K}^{[27]}$ and experimental data at $25 \mathrm{~K} \cdot{ }^{[28]}$ This shift is further evidence of temperature-induced vibrational softening in line with results by Steigmeier and Harbeke for GeTe. ${ }^{[28]}$ Such a phonon softening helps to understand the low thermal conductivity observed under ambient conditions.

To explain the vibrational softening of the optical modes upon crystallization, we start by summarizing the most important results of the structural investigations of the amorphous and crystalline phase. This summary will help to understand the bonding conditions that lead to the vibrational softening.

For the amorphous phase, four important conclusions can be derived from the data analysis. i) The bond lengths are considerably shorter than those in the crystalline phase, and ii) the co-ordination numbers are largely consistent with the 8-N rule (see Supporting Information for details). These first two observations are in line with the results of previous experimental studies. ${ }^{[25,29]}$ iii) The bond lengths only show a weak 
temperature dependence, and iv) the dependence of the atomic displacements on temperature is low. These findings provide clear evidence for covalent bonding in the amorphous state (hence the bond length is consistent with the sum of the atomic radii of the constituting atoms ${ }^{[30]}$ and the $8-\mathrm{N}$ rule is essentially satisfied), with only a very small anharmonic contribution to the interaction potential (hence the atomic spacing is largely independent of temperature) and relatively strong bonds between neighboring atoms. Therefore the temperature dependence of thermal displacements is small.

To facilitate the comparison between the amorphous and crystalline states, a list of structural properties that characterize the crystalline phase is compiled. This enumeration is in part based on Rietveld refinements of X-ray diffraction data for the crystalline phase measured at different temperatures. The number of nearest neighbors of each atom can be determined from the crystal structure (see Supporting Information). In the ideal rock-salt structure with a lattice constant of approximately $6 \AA$, the nearest neighbor spacing is $3 \AA$, while the second-nearest neighbor has a distance of approximately $4.25 \AA$. Hence all atoms that were closer than $3.5 \AA$ to the $\mathrm{Ge}$ atoms or $\mathrm{Sb}$ atoms, respectively, were counted as nearest neighbors of the corresponding $\mathrm{Ge}$ or $\mathrm{Sb}$ atom. With this definition, both the $\mathrm{Ge}$ and the $\mathrm{Sb}$ atoms have on average six nearest neighbors of Te. Clearly, these co-ordination numbers are not consistent with the $8-\mathrm{N}$ rule, which would predict that the $\mathrm{Ge}$ atoms have four nearest neighbors, while the $\mathrm{Sb}$ atoms should have three nearest neighbors. Furthermore, the interatomic spacing between the atoms is larger than the sum of the radii of the constituent atoms, as can be seen, e.g., for the Ge-Te bond lengths. This bond length amounts to $2.85 \AA$ in the crystalline rock-salt phase, while a significantly smaller value of $2.58 \AA$ is found in the amorphous phase. The latter value perfectly agrees with the sum of covalent radii $\left(2.58 \AA^{[31]}\right)$. In addition, the lattice constant of the crystalline phase shows a large thermal expansion of $\beta=5.05 \times 10^{-5} \mathrm{~K}^{-1}$. Then, there are significant static distortions in the metastable rock salt structure.

We observe three differences between the amorphous and the crystalline state. In comparison with the amorphous phase, the rock-salt-like structure of $\mathrm{Ge}_{2} \mathrm{Sb}_{2} \mathrm{Te}_{5}$ displays i) larger coordination numbers, ii) longer bond lengths, and iii) a large coefficient of thermal expansion. These differences imply that the bonding in the crystalline state is not ordinary covalent bonding as employed in the amorphous state. Instead, a remarkable variant of covalent bonding is utilized ${ }^{[9,11]}$ in the crystalline phase, which was coined 'resonance bonding' by Pauling. ${ }^{[32]}$ In phasechange materials such as GeTe or $\mathrm{Ge}_{1} \mathrm{Sb}_{2} \mathrm{Te}_{4}$, on average five valence electrons per atom are found. Since $\mathrm{sp}^{3}$ hybridization is unfavorable in this situation, bonding is promoted by the $p$ electrons, which leads to an octahedral-like co-ordination. ${ }^{\text {[3] }}$ This immediately explains the atomic arrangement in the crystalline phase. By employing valence-bond theory the larger interatomic spacing in the crystalline state can be elucidated. In this case, there are $3 \mathrm{p}$ electrons per atom available for six nearest neighbors, which gives rise to a bond order of 0.5 . This value is only half that for a single covalent bond utilized in the amorphous state. Since the bond order and the bond length are inversely related, this explains why, e.g., the distance between the Ge and the neighboring Te atom is significantly shorter in the amorphous state where the bond order is higher.

The litmus test for the relevance of resonance bonding is whether it can provide an explanation for the unconventional vibrational properties of the crystalline phase. As shown above, this state is characterized by smaller optical phonon energies yet a larger bulk modulus than the corresponding amorphous phase. Hence the question arises if these two observations can be explained by the unique bonding type in the crystalline phase. Resonance bonding is only possible in crystalline phasechange materials as it requires medium-range order which is missing in the amorphous phase ${ }^{[11]}$ This bonding mechanism has already been shown to have a profound influence on the electronic and optical properties of the crystalline state, where it leads to a smaller bandgap and a larger electronic polarizability than in the amorphous state. ${ }^{[1]}$ However, the mechanism also has a pronounced impact on the vibrational properties of the crystalline phase. Resonance bonding is accompanied by high values of the transverse effective charge $Z_{\mathrm{T}}{ }^{*}{ }^{* 33]}$ In the case of GeTe, this high value of $Z_{\mathrm{T}}{ }^{*}$ was found to increase further upon decreasing the rhombohedral distortion of the rock-salt-type phase. The relaxation towards the undistorted cubic phase is accompanied by a strong softening of the TO and LO modes as shown by Steigmeier and Harbeke. ${ }^{[28]}$ This result demonstrates the close link between resonance bonding and the vibrational properties of phase-change materials.

The low frequency TO phonons of the rock salt structure are closely related to the inherent tendency of the lattice to form a Peierls-like distortion. ${ }^{[34]}$ Indeed, the large static displacements are another fingerprint of crystalline phase-change materials. ${ }^{[9]}$ Hence the corresponding crystal can be best described by the potential shown in Figure 4. This potential is more anharmonic and weaker than the corresponding potential for the amorphous

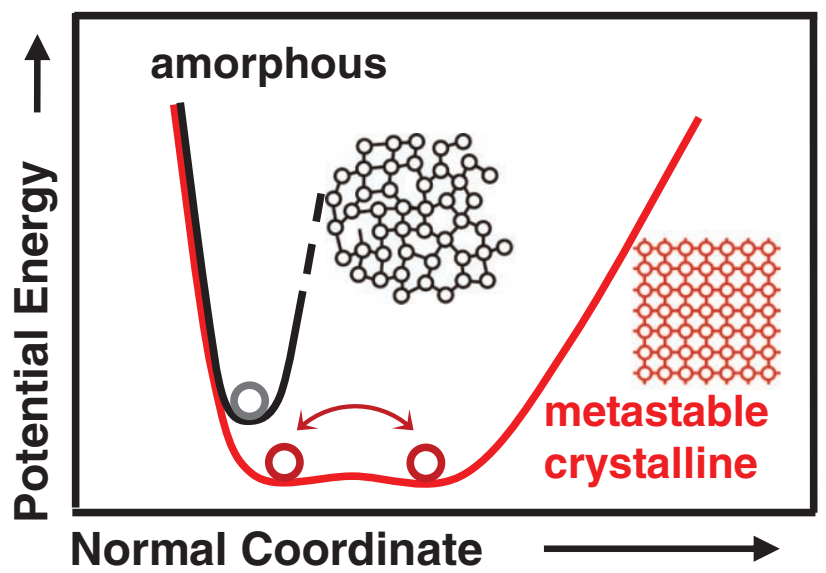

Figure 4. Schematic interaction potential for the amorphous and the crystalline state of a characteristic phase-change material such as $\mathrm{Ge}_{2} \mathrm{Sb}_{2} \mathrm{Te}_{5}$. The interaction potential for certain normal co-ordinates in the amorphous phase is more harmonic (symmetric), which results in smaller thermal expansion. The interaction potential of the metastable crystalline phase is weaker and more anharmonic, which leads to significant thermal expansion. The combination of resonance bonding and static distortions leads to this interaction potential, which explains a number of characteristic properties such as the pronounced anharmonicity and the soft phonon modes. 
state. It should be noted that such a potential form exists in the crystalline phase for a significant number of normal coordinates. In a recent calculation of the lattice dynamics of, e.g., $\mathrm{Ge}_{1} \mathrm{Sb}_{2} \mathrm{Te}_{4}$, Lencer observed an anharmonic shape of the potential for several normal co-ordinates. ${ }^{[35]}$

Finally, we will focus on another aspect: The relevance of the anharmonicity of the interaction potential and its consequences for the thermal properties. For $\mathrm{Ge}_{2} \mathrm{Sb}_{2} \mathrm{Te}_{5}$, e.g., the bulk modulus was determined by stress measurements ${ }^{[36,37]}$ and Kalb et al. identified a biaxial modulus of 28(5) GPa for the amorphous and 45(9) GPa for the crystalline phase, while Park et al. obtained values of 30(2) GPa and 37(2) GPa, respectively. Together with the measured coefficient of thermal expansion, the high temperature limit of the acoustic-mode Grüneisen parameter $\gamma^{[30]}$ can be evaluated to quantify the anharmonicity:

$\gamma=\frac{\beta K V_{\mathrm{m}}}{C_{\mathrm{V}}}$

In this formula, $\beta$ denotes the volume expansion, $\mathrm{C}_{V}$ is the specific heat capacity at constant volume that can be determined from the Dulong-Petit law, while $V_{\mathrm{m}}$ is the molar volume, which is obtained by XRD. From these numbers we determine a value of 1.5 for $\gamma$, if a bulk modulus $K$ of 41(2) GPa is used. ${ }^{[38]}$ This is a rather large value for $\gamma$, which demonstrates that phasechange materials in the crystalline state possess a rather anharmonic potential. A value of 1.5 is higher than the value found for most inorganic solids which frequently show values of $\gamma$ around 1 (e.g., for diamond 0.95, Si 0.96, Ge 1.00, GaAs $\left.1.11^{[39]}\right)$. Interestingly, related materials such as PbTe (1.45) and $\mathrm{AgSbTe}_{2}$ (2.05), ${ }^{[40]}$ which are also well-known thermoelectric materials, show similarly high values of $\gamma$ to $\mathrm{Ge}_{2} \mathrm{Sb}_{2} \mathrm{Te}_{5}$. $\mathrm{AgSbTe}_{2}$ has been previously shown to have characteristic features of phase-change materials ${ }^{[41]}$ such as an octahedral atomic arrangement and significant optical contrast between the amorphous and the crystalline state, as well as a high electronic polarizability in the crystalline state. ${ }^{[42,43]}$ Hence we can summarize that all the phase-change materials identified so far seem to possess anharmonic interaction potentials in the crystalline state.

The amorphous phase of $\mathrm{Ge}_{2} \mathrm{Sb}_{2} \mathrm{Te}_{5}$ has a significantly lower bulk modulus and a smaller coefficient of linear thermal expansion. This result is evident from stress measurements, ${ }^{[36,44]}$ which determine $\gamma$ to be $0.6(3)$ in the amorphous and 1.4(6) in the crystalline material. ${ }^{[36]}$ The latter value agrees well with the data point we have discussed above $(\gamma=1.5)$ which was obtained from our XRD measurements and elastic constants derived from DFT calculations for the crystalline phase. ${ }^{[45]}$

In the crystalline phase, the combination of a large bulk modulus and a large thermal coefficient of thermal expansion coefficient leading to a large anharmonicity contributes, together with the very low Debye temperature, to the extremely low thermal conductivity, which is proportional to the third power of the Debye temperature and inversely proportional to the anharmonicity $\gamma_{\cdot}^{[46]}$ These parameters hence contribute to the low thermal conductivity of $\mathrm{Ge}_{2} \mathrm{Sb}_{2} \mathrm{Te}_{5}$ of around $0.44 \mathrm{~W} \mathrm{~K}^{-1} \mathrm{~m}^{-1}$, while semiconductors with ordinary covalent bonding such as Si $\left(156 \mathrm{~W} \mathrm{~K}^{-1} \mathrm{~m}^{-1}\right),{ }^{[47]} \mathrm{Ge}\left(60 \mathrm{~W} \mathrm{~K}^{-1} \mathrm{~m}^{-1}\right),{ }^{[19]}$ or GaAs (45 W $\left.\mathrm{K}^{-1} \mathrm{~m}^{-1}\right)^{[20]}$ all have much higher thermal conductivity.

\section{Conclusions}

The picture provided here links the low thermal conductivity in crystalline phase-change materials with the prevalence of resonance bonding and the existence of lattice distortions in these chalcogenides. Recently a map was devised ${ }^{[9]}$ which helps to locate materials that display resonance bonding and possess atomic displacements away from a perfect octahedral arrangement. Hence this map also provides a blueprint to identify solids with low Debye temperatures, soft phonon modes, and anharmonic interactions, leading to an extremely low thermal conductivity of the lattice. This attribute of phase-change materials comes in very handy for applications. The low thermal conductivity ensures that the heat generated in the phase change layer upon melting is not transported away too rapidly, which would hence reduce the power needed to switch a crystalline phase change region to the amorphous state. We note in passing that a potential with only resonant bonds but no distortions would not lead to the desired thermal properties since in this case the potential is more harmonic and stronger, more closely resembling the metallic state. Hence it is the combination of resonance bonding and local lattice distortions which characterize the structure and bonding of crystalline phase-change materials that can explain many of the essential and characteristic properties of this material class.

\section{Experimental Section}

XRD: The specimen for the PDF and Rietveld analyses of the crystalline phase was prepared by the following method. First, a thin film of $\mathrm{Ge}_{2} \mathrm{Sb}_{2} \mathrm{Te}_{5}$ about 300-nm thickness was sputtered onto a glass disk of 120-mm diameter. To prevent oxidation, a 2-nm thin $\mathrm{SiO}_{2}$ passivation layer was sputtered onto this film. The film was amorphous just after its formation. It was crystallized by laser irradiation into a metastable phase. The completed film was then, just before the measurement, powdered by scraping with a spatula, and the powder was packed in a quartz capillary tube with an internal diameter of $0.2 \mathrm{~mm}$. To insulate against the atmosphere, the opening of the capillary was sealed with an oxyacetylene flame. The diffraction experiments were performed using beamline BL02B2 at the Japan Synchrotron Radiation Research Institute (SPring-8). ${ }^{[8]}$ The energy of the incident beam was about $29.3 \mathrm{keV}$; intensity data were collected using a Debye-Scherrer camera. High- and low-temperature experiments were also performed while nitrogen gas was blown onto the capillary tube, set to specified temperatures. The temperatures were calibrated in advance by using a type-R thermocouple placed just at the position of the specimen (capillary) to be measured.

EXAFS: The amorphous specimens for EXAFS experiments were prepared by laminating organic film sheets onto glass disks with a diameter of $120 \mathrm{~mm}$ and sputter deposition to form the amorphous films with a thickness of $200 \mathrm{~nm}$. A 2-nm thin $\mathrm{SiO}_{2}$ passivation layer was also sputtered onto these films to prevent oxidation. The organic film sheets were peeled from the glass disks and cut into square pieces about $10 \mathrm{~mm} \times 10 \mathrm{~mm}$ in size; appropriate numbers of sheet were then stacked to form measurement specimens. The EXAFS measurements were carried out at the BL01B1 beam line of SPring-8. The incident X-ray beam was monochromatized by a Si double crystal using the (111) net planes. Contamination with higher harmonics in the incident beam was 
removed by means of Pt-coated mirrors located downstream of the monochromator. EXAFS data of $\mathrm{Ge}, \mathrm{Sb}$, and Te K-edges were collected in transmission mode and the temperature of the specimen was controlled by an electric furnace and a cryostat furnace.

NIS: The samples for nuclear inelastic-scattering measurements were prepared by thermal evaporation on aluminum foil. The evaporation was performed from three independent, single-elemental Knudsen cells, filled with $\mathrm{GeTe}$ and $\mathrm{Sb}$ in natural abundance and with ${ }^{125} \mathrm{Te}$, which led to a $77 \%$ isotopic concentration of ${ }^{125} \mathrm{Te}$ in the final sample of $\mathrm{Ge}_{1} \mathrm{Sb}_{2} \mathrm{Te}_{4}$. The measurements were carried out with the samples at $25 \mathrm{~K}$ to minimize multiphonon contributions. The high-resolution backscattering monochromator used the (8 $16-2440)$ and $(91-1068)$ reflections of sapphire and had an energy resolution of 1.4 and $1.1 \mathrm{meV}$ for the $37.1 \mathrm{keV}^{121} \mathrm{Sb}^{[49]}$ and $35.5 \mathrm{keV}^{125} \mathrm{Te}^{[50]}$ nuclear resonances, respectively. The resolution employed in the present study was significantly enhanced over that employed in recent reports in the literature. ${ }^{[49,50]}$ The consistence of the obtained atomic mean-square displacements and force constants was verified by comparing the values obtained directly from the spectral distribution and from the density of states. ${ }^{[24]}$ After performing the NIS measurements, we verified by means of XRD that the samples were indeed in the amorphous and $\mathrm{NaCl}$-type phase.

\section{Supporting Information}

Supporting Information is available from the Wiley Online Library or from the author.

\section{Acknowledgements}

Some synchrotron radiation experiments were performed on BL01B1 and BL02B2 at SPring-8 with the approval of the Japan Synchrotron Radiation Research Institute (JASRI). We express our sincere gratitude to Dr. K. Kato and Dr. K. Osaka at JASRI for their assistance with the experiment. We also express our sincere thanks to Mr. Katsumi Kawahara at Panasonic for his precise preparations of Ge-Sb-Te film samples. The structural model in Figure 4 of the Supporting Information was displayed using Java Structure Viewer (JSV 1.08 lite), created by Dr. Steffen Weber. Financial support by the Deutsche Forschungsgemeinschaft (Wu 243/17) and from the Helmholtz Gemeinschaft Deutscher Forschungszentren (RH Young Investigator Group) is gratefully acknowledged. We thank the European Synchrotron Radiation Facility for provision of nuclear inelastic-scattering beamtime at beamline ID22N and Mr. D. Bessas for his assistance during the experiment. A part of this work was supported by Core Research for Evolutional Science and Technology (CREST) "X-ray pinpoint structural measurement project: Development of the spatial- and time-resolved structural study for nano-materials and devices" and by the Academy of Finland and the Japan Science and Technology Agency through the Strategic Japanese-Finnish Cooperative Program on "Functional materials".

Received: October 13, 2010

Revised: January 25, 2011 Published online: April 26, 2011

[1] M. Wuttig, N. Yamada, Nature Materials 2007, 6, 824.

[2] N. Yamada, E. Ohno, K. Nishiuchi, N. Akahira, M. Takao, J. Appl. Phys. 1991, 69, 2849.

[3] S. R. Ovshinsky, Phys. Rev. Lett. 1968, 21,1450.

[4] R. Bez, Microelectron. Eng. 2005, 80, 249.

[5] G. Bruns, P. Merkelbach, C. Schlockermann, M. Salinga, M. Wuttig, T. D. Happ, J. B. Philipp, M. Kund, Appl. Phys. Lett. 2009, 95, 3108.

[6] I. Friedrich, V. Weidenhof, W. Njoroge, P. Franz, M. Wuttig, J. Appl. Phys. 2000, 87, 4130.

[7] W. P. Risk, C. T. Rettner, S. Raoux, Appl. Phys. Lett. 2009, 94, 1906.
[8] W. Welnic, S. Botti, L. Reining, M. Wuttig, Phys. Rev. Lett. 2007, 98, 6403.

[9] D. Lencer, M. Salinga, B. Grabowski, T. Hickel, J. Neugebauer, M. Wuttig, Nature Materials 2008, 7, 972.

[10] B. Huang, J. Robertson, Phys. Rev. B 2010, 81, 1204.

[11] K. Shportko, S. Kremers, M. Woda, D. Lencer, J. Robertson, M. Wuttig, Nature Materials 2008, 7, 653.

[12] T. Siegrist, P. Jost, H. Volker, M. Woda, C. Schlockermann, P. Merkelbach, M. Wuttig, Nature Materials 2011, 10, 202.

[13] J. P. Reifenberg, M.A. Panzer, S. Kim, A. M. Gibby, Y. Zhang, S. Wong, H. S. P. Wong, E. Pop, K. E. Goodson, Appl. Phys. Lett. 2007, 91, 1904.

[14] H. K. Lyeo, D. G. Cahill, B. S. Lee, J. R. Abelson, M. H. Kwon, K. B. Kim, S. G. Bishop, B. K. Cheong, Appl. Phys. Lett. 2006, 89, 151904.

[15] C. Peng, M. Mansuripur, Appl. Opt. 2002, 41, 361.

[16] V. Giraud, J. Cluzel, V. Sousa, A. Jacquot, A. Dauscher, B. Lenoir, H. Scherrer, S. Romer, J. Appl. Phys. 2005, 98, 3520.

[17] E. Kim, S. Kwun, S. M. Lee, H. Seo, J. G. Yoon, Appl. Phys. Lett. 2000, 76, 3864.

[18] E. R. Meinders, C. Peng, J. Appl. Phys. 2003, 93, 3207.

[19] S.R. Bakhchieva, N. P. Kekelidze, M. G. Kekua, Phys. Status Solidi A 1984, 83, 139.

[20] E. F. Steigmeier, I. Kudman, Phys. Rev. 1963, 132, 508.

[21] M. G. Holland, Phys. Rev. 1964, 134, A471.

[22] P. Nath, K. L. Chopra, Phys. Rev. B 1974, 10, 3412.

[23] G. Dalba, P. Fornasini, J. Synchrotron Radiat. 1997, 4, 243.

[24] R. Rüffer, A. I. Chumakov, Hyperfine Interact. 2000, 128, 255.

[25] P. Jóvári, I. Kaban, J. Steiner, B. Beuneu, A. Schöps, M. Webb, Phys. Rev. B 2008, 77, 035202.

[26] G. C. Sosso, S. Caravati, M. Bernasconi, J. Phys. Condens. Matter 2009, 21, 095410.

[27] R. Shaltaf, X. Gonze, M. Cardona, R. Kremer, G. Siegle, Phys. Rev. B 2009, 79, 1.

[28] E. F. Steigmeier, G. Harbeke, Solid State Commun. 1970, 8, 1275.

[29] D. Baker, M. Paesler, G. Lucovsky, S. Agarwal, P. Taylor, Phys. Rev. Lett. 2006, 96, 5 .

[30] K. Hirota, K. Nagino, G. Ohbayashi, J. Appl. Phys. 1997, 82, 65.

[31] B. Cordero, V. Gómez, A. E. Platero-Prats, M. Revés, J. Echeverría, E. Cremades, F. Barragán, S. Alvarez, Dalton Trans. 2008, 2832.

[32] L. Pauling, Nature Of the Chemical Bond, Cornell Univ. Press, New York 1939.

[33] G. Lucovsky, R. White, Phys. Rev. B 1973, 8, 660.

[34] P. Littlewood, Crit. Rev. Solid State Mater. Sci. 1983, 11, 229.

[35] D. Lencer, Design-Rules, Local Structure and Lattice-Dynamics of Phase-Change Materials For Data Storage Applications, Doctoral Dissertation, RWTH Aachen, 2011.

[36] J. Kalb, F. Spaepen, T. P. Leervad Pedersen, M. Wuttig, J. Appl. Phys. 2003, 94, 4908.

[37] I. Park, J. Jung, S. Ryu, K. Choi, B. Yu, Y. Park, S. Han, Y. Joo, Thin Solid Films 2008, 517, 848.

[38] A. V. Kolobov, J. Haines, A. Pradel, M. Ribes, P. Fons, J. Tominaga, Y. Katayama, T. Hammouda, T. Uruga, Phys. Rev. Lett. 2006, 97, 035701-1.

[39] S. Adachi, Properties Of group-IV, III-V and II-VI Semiconductors, John Wiley \& Sons Ltd., Chichester 2005.

[40] D. T. Morelli, V. Jovovic, J. P. Heremans, Phys. Rev. Lett. 2008, 101, 035901.

[41] R. Detemple, D. Wamwangi, M. Wuttig, G. Bihlmayer, Appl. Phys. Lett. 2003, 83, 2572

[42] H. A. Zayed, A. M. Ibrahim, L. I. Soliman, Vacuum 1996, 47, 49.

[43] L. H. Ye, K. Hoang, A. Freeman, S. Mahanti, J. He, T. Tritt, M. Kanatzidis, Phys. Rev. B 2008, 77, 1.

[44] T. P. Leervad Pedersen, J. Kalb, W. K. Njoroge, D. Wamwangi, M. Wuttig, F. Spaepen, Appl. Phys. 2001, 79, 3597. 
[45] S. Caravati, M. Bernasconi, T. D. Kühne, M. Krack, M. Parrinello, J. Phys. Condens. Matter 2009, 21, 5501

[46] D. T. Morelli, J. P. Heremans, Appl. Phys. Lett. 2002, 81, 5126.

[47] C. J. Glassbrenner, G. A. Slack, Phys. Rev. 1964, 134, 1058.

[48] E. Nishibori, M. Takata, K. Kato, M. Sakata, Nucl. Instrum. Methods In Phys. Res. A 2001, 467-468, 1045.

[49] H. C. Wille, R. P. Hermann, I. Sergueev, O. Leupold, P. V. D. Linden, B. C. Sales, F. Grandjean, G. J. Long, R. Rüffer, Y. V. Shvyd'ko, Phys. Rev. B 2007, 76, 140301.
[50] H. C. Wille, R. P. Hermann, I. Sergueev, U. Pelzer, A. Möchel, T. Claudio, R. Rüffer, A. Said, Y. V. Shvyd'ko, Europhys. Lett. 2010, $91,62001$.

[51] R. Mazzarello, S. Caravati, S. Angioletti-Uberti, M. Bernasconi, M. Parrinello, Phys. Rev. Lett. 2010, 104, 1.

[52] P. Zalden, C. Bichara, J. van Eijk, C. Braun, W. Bensch, M. Wuttig, J. Appl. Phys. 2010, 107, 4312.

[53] S. Caravati, M. Bernasconi, T. D. Kühne, M. Krack, M. Parrinello, J. Phys. Condens. Matter 2009, 21, 255501. 